\title{
Phosphatidylcholine to Albumin Ratio Measurement
}

National Cancer Institute

\section{Source}

National Cancer Institute. Phosphatidylcholine to Albumin Ratio Measurement. NCI

Thesaurus. Code C147420.

The determination of the ratio of phosphatidylcholine compared to albumin present in a sample. The measurement may be expressed as a ratio or percentage. 